\title{
LO QUE EL VIENTO SE LLEVÓ: NO PARTICIPACIÓN LOCAL EN UN CONFLICTO SOBRE UN AMBIENTE DE DUNAS
}

\author{
Patricio Bruno Besana ${ }^{\star}$ \\ https://orcid.org/0000-0003-0757-0121 \\ Soledad Fernández Bouzo ${ }^{*}$ \\ hitps://orcid.org/0000-0003-2817-489X \\ Ana Laura Monserrat ${ }^{* *}$
}

RECIBIDO: Diciembre 2018 / ACEPTADO: Agosto 2019 / PUBLICADO: Enero 2020

\begin{abstract}
Como citar: Besana, Patricio; Fernández Bouzo, Soledad; Monserrat, Ana. (2020). Lo que el viento se llevó: No participación local en un conflicto sobre un ambiente de dunas. Telos: revista de Estudios Interdisciplinarios en Ciencias Sociales, 22 (1), Venezuela. (Pp.6-30).

DOI: www.doi.org/10.36390/telos221.02
\end{abstract}

\section{RESUMEN}

El presente trabajo propone a la Teoría del Actor Red (TAR), en particular a la perspectiva de Michel Callon (1986, 2001), como encuadre teórico metodológico para el análisis de conflictos ambientales desde un enfoque interprofesional que permite enriquecer el conocimiento de las ciencias sociales a partir de conocimientos provenientes de las ciencias naturales. Su objetivo es brindar una explicación posible sobre los factores que inciden en la falta de participación de actores locales en conflictos ambientales. En adición a la TAR, para ello se apela a un análisis desde la literatura latinoamericana de los conflictos ambientales (con énfasis en autores y obras como: Gudynas y Acosta, (2011); Svampa, (2012) y Gutiérrez e Isuani, (2014); entre otros. Se argumenta que las características del ambiente de dunas en cuestión inciden en la no participación de la población local. Para llevar a cabo el análisis se desarrollaron observaciones de flora y fauna, entrevistas semi-estructuradas a pobladores y autoridades de la zona, entrevistas a informantes clave, análisis de documentos públicos y notas periodísticas.

Palabras clave: Ambiente de dunas; Conflictos socio-ambientales; No participación; Perspectiva interprofesional; Teoría del Actor Red.

\footnotetext{
* Es Doctor en Ciencia Política y Licenciado en Sociología. Se desempeña como Docente y Becario Postdoctoral en la Escuela de Política y Gobierno de la Universidad Nacional de San Martín (UNSAM). Es miembro del Grupo Ambiente y Política (GAP). Ha publicado artículos, libros y capítulos sobre ambiente, participación política, políticas públicas y pobreza en Argentina, Chile, Colombia, España, México, Uruguay y Venezuela. Argentina. Correo electrónico: pbesana@unsam.edu.ar

"Es Doctora en Ciencias Sociales y Licenciada en Sociología. Es investigadora del CONICET con sede en el Área de Estudios Urbanos del Instituto de Investigaciones "Gino Germani", donde forma parte del Grupo de Estudios Ambientales. Realizó videos de investigación que versan sobre los procesos de participación social en la recomposición ambiental de la cuenca Matanza-Riachuelo. Es directora de Quid 16, revista del Área de Estudios Urbanos del IIGG. Se desempeña como docente de grado y posgrado en asignaturas que versan sobre los temas que investiga. Argentina. Correo electrónico: soledad.fernandezbouzo@gmail.com

"** Es Doctora y Licenciada en Ciencias Biológicas, especialista en ecología de dunas costeras. Se desempeña como Docente en la Universidad Favaloro y como Acompañante didáctica en el Ministerio de Educación de la Nación Argentina. Ha publicado artículos sobre su especialidad en numerosos países. Argentina. Correo electrónico: monserrat.giunta@gmail.com
} 


\section{Gone with the wind: Local non participation and conflict on a dune environment}

\section{ABSTRACT}

This paper proposes the actor network theory (ANT), in particular the Michel Callon's perspective $(1986,2001)$, for the analysis of environmental conflicts as a theoretical and methodological frame of an interprofessional approach that uses knowledge of the natural sciences to enrich knowledge of social sciences. The present aims to bring a possible explanation of the lack of participation of local people on environmental conflicts. In addition to the ANT, it is used LatinAmerican literature in particular authors as: Gudynas y Acosta, (2011); Svampa, (2012) and Gutierrez e Isuani, (2014); amongs others. It is argued that the characteristics of the dune environment affect the non-participation of the local population. To this end, observations were made of flora and fauna, semi-structured interviews with local residents and authorities, interviews with key informants and analysis of public documents and journalistic notes.

Keywords: Dune environment, Environmental conflicts, Interprofessional approach, Non participation, Actor Network Theory.

\section{Introducción}

El creciente interés científico que despierta el ambiente suscita constantes conflictos y controversias entre disciplinas, expertos y actores territoriales a lo largo del mundo (Azuela, 2006; Gutiérrez, 2015). En ciencias sociales, gran parte de la literatura ambiental ha hecho de dichos conflictos y controversias su objeto de estudio (Harvey, 1996; Hajer, 1997; Sabatini, 1997; Sousa Santos, 2001; Tischler, 2004; Acselrad, 2006; Alimonda, 2006; Leff, 2006; Gudynas y Acosta, 2011; Reboratti, 2012; Svampa, 2012; entre otros). En el norte global, buena parte de dicha literatura puso el acento en las controversias entre expertos y disciplinas científicas en torno al ambiente o hizo énfasis en la combinación entre controversias expertas y participación de actores locales no expertos (Harvey, 1996; Hajer, 1997; Fischer, 2000). Debajo del Ecuador, el panorama parece más diverso.

A las preocupaciones de los estudios del norte, se agregan las de otros trabajos, generalmente, afines a la literatura crítica latinoamericana-que enfatizan el modo en que los saberes de actores de la región disputan y construyen sentido frente a los fundamentos de disciplinas científicas y modelos de acumulación que conciben al "ambiente" o a la "naturaleza" meramente como un recurso a explotar. (Harvey, 1996; Hajer, 1997; Sabatini,1997; Fischer, 2000; Sousa Santos, 2001; Tischler, 2004; Acselrad, 2006; Alimonda, 2006; Leff, 2006; Alcañiz y Gutiérrez, 2009; Tischler, 2010; Gudynas y Acosta, 2011; Melé 2006; Svampa, 2012, entre otros).

En ese contexto, el presente trabajo estudia un conflicto ambiental poco común para la región, entre otras cosas, por carecer precisamente de participación de actores locales. Si bien, éste se desarrolla entre dos coaliciones de expertos por los valores y usos atribuidos a un ambiente de dunas ubicado en el Partido de Coronel Dorrego, región costera austral de la 
Provincia de Buenos Aires, Argentina (analizado en profundidad en otros trabajos de los autores - Besana y Monserrat, 2013 y Besana, 2018); interesa analizar aquí las características que lo hacen distinto de la mayoría de los conflictos estudiados por la literatura latinoamericana. Dichas características incluyen: el ambiente de dunas donde tiene lugar el conflicto (distinto de los espacios típicamente urbanos o rurales donde suelen desarrollarse los casos analizados por gran parte de la literatura ambiental latinoamericana- Eg. Sabatini,1997; Giarracca, 2001; Reboratti, 2012); y la mencionada falta de participación de la población local.

Para desarrollar dicho análisis se optó por una estrategia doble que incorpora un repaso de la literatura latinoamericana sobre conflictos ambientales, así como la apelación a un enfoque interprofesional (Eg. Hammic et al., 2007). La revisión de literatura tuvo por objeto situar en el contexto de América Latina, en particular en el de Argentina, al conflicto analizado. Entre otras cosas, en el apartado dedicado a ello se sugiere que la impronta general con que se institucionalizaron las agencias ambientales estatales en Argentina, mayormente vinculadas a la promoción de proyectos y políticas ligadas a la agenda ambiental global (Gutiérrez e Isuani, 2014), se relaciona con la emergencia del conflicto en cuestión, aunque no alcanza por sí sola para explicarlo.

El enfoque interprofesional, por su parte, se implementa con el fin de enriquecer el análisis de las ciencias sociales mediante la incorporación de conocimientos de las ciencias naturales (especialmente de la biología). Este enfoque aporta una explicación posible sobre la falta de participación de actores locales a la que no podría arribarse de no contar con los aportes de la biología. Para llevar a cabo dicho enfoque interprofesional, se considera el uso de categorías analíticas de la Teoría del Actor Red (TAR de aquí en más -Callon, 1986; Latour, 2007), en tanto facilita el diálogo entre ciencias sociales y naturales al incorporarindistintamente- en su análisis a entidades humanas y no humanas. En consecuencia, a partir de la apelación a un enfoque interprofesional, se argumenta que la falta de participación de la población local en el conflicto se relaciona, en gran medida, con su desconocimiento y desinterés para con el ambiente de dunas como resultado de algunas características de este último.

En suma, el artículo se organiza del siguiente modo: el próximo apartado sitúa al conflicto en el contexto latinoamericano y argentino. En el que sigue, se desarrolla la metodología utilizada. A continuación, se ubica al Partido de Coronel Dorrego y se describen algunas características del ambiente de dunas donde se desarrolla el conflicto. En el apartado siguiente, se repasa cómo dos coaliciones de expertos entran en conflicto por los usos que pretenden asignarle al ambiente de dunas. En el apartado que le sigue, se presentan las categorías analíticas de la TAR utilizadas para desarrollar el argumento presentado. Acto seguido, se hace uso de dichas categorías para analizar cómo ciertas características del ambiente de dunas incidieron en la no participación de grupos de la población local. Por último, el artículo finaliza con una serie de preguntas y reflexiones. 


\section{Conflictos ambientales en América Latina e institucionalización del ambiente en el Estado} Argentino.

Desde tiempos coloniales, los bienes naturales de América Latina son vistos como recursos económicos a explotar por parte de Estados y privados (vg. Leff, 2006; Svampa, 2012). Esto supuso diversas dinámicas de desposesión y despojo de tierras, bienes comunes y territorios (Harvey, 1996) y motivó, consecuentemente, procesos de resistencias sociales por parte de actores y comunidades locales (Hajer, 1997; Sabatini, 1997; Sousa Santos, 2001; Tischler, 2004; Acselrad, 2006; Alimonda, 2006; Leff, 2006; Alcañiz y Gutiérrez, 2009; Tischler, 2010; Gudynas y Acosta, 2011; Melé, 2006;Svampa, 2012; Christel, 2018; Figueroa y Gutiérrez, 2018; Montera et al, 2018; Novas, 2018; entre otros). Dichas dinámicas, así como los procesos de resistencias que suscitaron, se desarrollaron con más o menos fuerza en distintas etapas de la historia reciente de la región, a raíz de factores domésticos e internacionales. Asimismo, los conflictos que ocasionaron dichas dinámicas opusieron discursos y formas globales de ponderar al ambiente frente a concepciones locales y a formas tradicionales de reproducción de la vida doméstica (Martínez Allier, 1991; Souza Santos, 2001; Tischler, 2004; Tischler, 2010; Gudynas y Acosta, 2011).

El modelo de acumulación basado en la explotación de bienes primarios se inscribió en un contexto global y contó con discursos afines y categorías universalistas que lo justificaron -tales como modernidad, progreso y desarrollo (Souza Santos, 2001). En ese contexto, el auge del positivismo y de las ciencias occidentales a lo largo del mundo fue funcional a las elites latinoamericanas que cooptaron las estructuras de los jóvenes Estados Nacionales. Históricamente, dichas elites echaron las culpas por las fallas del modelo de acumulación basado en la explotación de bienes primarios a sujetos y comunidades autóctonas, sus culturas y categorías; consideradas "rémoras" del pasado (Vg. Sarmiento, 2009).

Frente a ello, hacia las décadas de 1950, 1960 y 1970, comenzaron a acumularse experiencias (fenómenos políticos, discursos y teorías) que ponían en tela juicio a las nociones mencionadas, presentaban versiones críticas de las mismas y/o recuperaban el legado de sujetos y tradiciones autóctonas. Posteriormente, conocidas como "alternativas al desarrollo" 0 "desarrollos alternativos" (Gudynas y Acosta, 2011; Svampa, 2012), muchas de dichas experiencias incluyeron nociones que consideran a las distintas formas de la naturaleza dentro de una concepción ampliada de comunidad.

Los violentos regímenes autoritarios que azotaron a gran parte de América Latina a partir de 1970 y las consecuentes reformas de mercado de inspiración neoliberales de las décadas de 1980 y 1990 pusieron en interregno a dicha "acumulación de experiencias" (Gudynas y Acosta, 2011; Svampa, 2012). La década de 1990 se caracterizó por el ascenso de gobiernos populares de derecha que, en línea con el Consenso de Washington, centraron sus modelos económicos principalmente en la valorización financiera (Svampa, 2012). 
El desgaste que produjeron las políticas públicas implementadas por dichos gobiernos trajo, al inicio del nuevo milenio, la emergencia de la nueva izquierda latinoamericana -también conocida como progresismo latinoamericano. Los nuevos gobiernos compartieron, en mayor 0 menor medida, el rechazo a la política económica neoliberal, la defensa del protagonismo del Estado, la satisfacción de demandas de sectores populares -generalmente urbanos- y la toma de medidas a favor de una distribución más equitativa de la riqueza (Svampa, 2012).

En países con una fuerte composición poblacional de pueblos originarios, diversos discursos fueron amalgamando elementos de las visiones críticas de las décadas pasadas con saberes tradicionales, comunitarios, campesinos e indígenas que -entre otras cuestionespusieron el acento en la necesidad de tener una relación más respetuosa con la naturaleza y el ambiente (Acselrad, 2006; Alimonda, 2006; Leff, 2006; Gudynas y Acosta, 2011). Dichos discursos dieron visibilidad y fortalecieron las posiciones y luchas de sujetos y comunidades locales, organizaciones de base, campesinos, pueblos originarios, entre otros; por sus lugares de origen (Acselrad, 2006; Alimonda, 2006; Leff, 2006; Gudynas y Acosta, 2011). No obstante, al mismo tiempo un pronunciado aumento en la demanda internacional de bienes primarios reintrodujo fuertemente el incentivo a la explotación y exportación de los mismos a gran escala. En conjunto, ambas cuestiones redundaron en un pronunciado aumento de la conflictividad ambiental en la región (Svampa, 2012). Actualmente, dicho incremento se mantiene en algunos países y se ha agravado en otros por el ascenso de gobiernos -generalmente de derecha- que implementan estrategias represivas y punitivas frente a las protestas sociales.

En Argentina, particularmente, el factor internacional no sólo influyó en el modelo de explotación de bienes primarios y en las demandas socio-ambientales que suscitó. Más recientemente, también lo hizo en el proceso de creación de instituciones ambientales en la esfera del Estado Nacional (Gutiérrez e Isuani, 2014). En ese sentido, varios hitos de la institucionalización del ambiente en la esfera pública darían cuenta de ello, tales como: a)la creación de la primera agencia ambiental estatal nacional en 1973 - durante la tercera presidencia de Juan Domingo Perón (1973 - 1976)-tan sólo un año después de la Conferencia de Estocolmo conocida como la primera cumbre de la Tierra; y b) el regreso del "ambiente" al primer plano de la institucionalidad del Estado Argentino en la década de 1990 (Gutiérrez e Isuani, 2014), tras una fase de retroceso durante el gobierno de facto que tuvo lugar entre 1976 y 1983.

Sobre esta segunda etapa de institucionalización del ambiente, varios autores consideran que la creación de la Secretaría de Recursos Naturales y Ambiente Humano de la Nación en 1991 -rebautizada Secretaría de Recursos Naturales y Desarrollo Sostenible en 1996, y la inclusión del derecho a un ambiente sano, entre otros, derechos ambientales de tipo procedimental en la carta magna de 1994, respondió primordialmente a la decisión del entonces presidente Menem de adherir a la agenda de Estados Unidos y de Organismos Internacionales, 
con la firme intención de acceder a distintas líneas de financiamiento internacional (Eg. Gutiérrez e Isuani, 2014).

En ese contexto, y más allá de los cambios que sufrió la institucionalidad ambiental en Argentina desde entonces, las agencias ambientales nacionales, y en buena medida las provinciales, tendieron a concebir al ambiente desde una perspectiva global. Dicha perspectiva, afín al concepto de desarrollo sostenible por tratar de reconciliar la idea de crecimiento económico con la de cuidado ambiental (Harvey, 1996), choca a menudo con los modos en que actores autóctonos conciben a la naturaleza y el ambiente local en el que desarrollan sus vidas. Aunque con menos recurrencia, la perspectiva global generalmente adoptada por las instituciones ambientales del Estado Argentino (Gutiérrez e Isuani, 2014), también enfrenta resistencias provenientes del propio entramado estatal o de coaliciones que incluyen tanto a actores de la sociedad civil como estatales (Eg. Christel, 2018; Figueroa y Gutiérrez, 2018; Montera et al, 2018; Novas, 2018); tal como el conflicto que aquí nos ocupa. En buena medida, éste resulta de la promoción delos Mecanismos de Desarrollo Limpio (de aquí en adelante MDLNaciones Unidas 1998), asociados a la mencionada perspectiva global del Estado, en un territorio que también fuera propuesto como Área de Natural Protegida (de aquí en más ANP).

En otros trabajos (Besana y Monserrat, 2013; Besana, 2018), se analizó en profundidad cómo el conflicto se desarrolló entre dos coaliciones integradas exclusivamente por expertos de las esferas del Estado y de la sociedad civil y cómo, en consecuencia, una visión "macro" del Estado Argentino tendiente a promover la sobreexplotación de recursos naturales y/o a institucionalizar la agenda ambiental global en su entramado de agencias no alcanza para explicar la emergencia de ciertos conflictos. Por el contrario, en línea con una perspectiva más micro y coalicional sobre políticas ambientales (Gutiérrez, 2018), en dichos trabajos se señaló cómo no es la pertenencia de los expertosa la esfera del Estado o a la de la sociedad civil lo que los une o enfrenta, sino el valor que asignan al espacio disputado, en relación a diversos discursos sobre el ambiente(Harvey, 1996; Hajer, 1997) que ponderan de formas divergentes las relaciones desarrollo económico/conservación, ser humano/naturaleza y escala local/escala global (Besana y Monserrat, 2013; Besana, 2018).

En este trabajo en particular, más que el conflicto entre expertos, interesa analizar los motivos que pudieron incidir en la falta de participación de actores locales. Puesto que el argumento que da cuenta de ello se relaciona con las características del ambiente de dunas disputado, y su influencia en la población local y la comunidad experta, a continuación, se presentan las características del enfoque interprofesional que permitieron aportar una explicación a partir de la conjunción entre conocimientos de las ciencias sociales y de las naturales. 


\section{Materiales y Métodos}

El enfoque interprofesional aquí propuesto se utilizó por primera vez en el área de las ciencias médicas (Vg. Hammic et al., 2007). Originalmente, dicho enfoque recurrió al uso de conocimientos y herramientas provenientes de las ciencias sociales con el fin de arribar a un buen diagnóstico en enfermedades cuyos orígenes se encuentran vinculados, principalmente, a hábitos sociales (Vg. Hammic et. al., 2007).

Sin alterar los principios del enfoque interprofesional, los aportes de este trabajo tienden a enriquecer el conocimiento de las ciencias sociales, a partir de conocimientos de las ciencias naturales -en especial del sub-campo de la ecología, perteneciente a las ciencias biológicas. Propio del interés de las ciencias sociales, el interrogante que pretende resolver este trabajo refiere a los factores que inciden en la falta de participación de actores locales en conflictos ambientales.

No obstante, la inquietud que da origen al enfoque interprofesional presentado en este trabajo parte de las dificultades sufridas por integrantes de una de las coaliciones de expertos en disputa a la hora de interesar la población local en las características del ambiente en cuestión. Dicha inquietud supuso una serie de colaboraciones entre científicos naturales y sociales, entre las que se encuentra éste y otros trabajos que lo anteceden ( $\mathrm{Vg}$. Besana y Monserrat, 2013). En consonancia con el enfoque interprofesional, el diseño metodológico propuesto aquí consta de un bricolaje de métodos múltiples (Denzin y Lincoln, 1994) que incluyen: observaciones de flora y fauna, análisis de documentos oficiales, entrevistas a protagonistas e informantes clave y revisión de notas periodísticas.

Las observaciones de flora y fauna fueron desarrolladas antes y durante el desarrollo del conflicto en cuestión (entre los años 2007 y 2009) en el marco de estudios biológicos. Las restantes herramientas y análisis se realizaron con posterioridad. Una primera campaña de entrevistas a vecinos se realizó a lo largo del año 2009, ésta fue reforzada mediante entrevistas a integrantes de las organizaciones en disputa, funcionarios públicos y vecinos en otras dos campañas: entre 2011 y 2013 y durante el año 2016.Las entrevistas a informantes clave se desarrollaron mayormente durante la última campaña, con objeto de comprender las lógicas generales con las que actúan determinadas instituciones.

En total, se realizaron 52 entrevistas distribuidas del siguiente modo: 30 a vecinos, 15 a integrantes y a funcionarios de las organizaciones y áreas del Estado en disputa y siete a informantes clave. Las entrevistas se desarrollaron siguiendo lineamientos de la perspectiva de la teoría fundamentada (cuyo fin es obtener categorías emergentes- Glaser y Strauss 1967). Acorde a dicha perspectiva, el tamaño de la muestra de entrevistas no se determinó a priori en base a criterios estadísticos, sino a partir del criterio de saturación teórica (Glaser y Strauss, 1967). Como ya es de público conocimiento, este criterio indica que la muestra se da por finalizada una vez que la realización de nuevas entrevistas ya no agrega nueva información (es decir, saturan teóricamente la muestra). En el presente, se citaron 18 de las 52 entrevistas 
realizadas -también en base al criterio de saturación teórica- por considerarse que las primeras grafican cabalmente la información contenida en el conjunto de las entrevistas.

Partido de Coronel Dorrego: Localización, población y ambiente de dunas

El Partido de Coronel Dorrego se encuentra al sudeste de la Provincia de Buenos Aires, en la denominada Región Costera Austral. Para el año 2010 el distrito contaba con una población estable de 15.825 habitantes (INDEC 2010). La ciudad cabecera, del mismo nombre que el distrito, es sede de la administración municipal. Las restantes localidades son eminentemente rurales, con excepción de la localidad de Marisol que constituye la única villa balnearia del distrito (Municipio de Coronel Dorrego, 2011), y es donde se desarrolla el conflicto.

\section{Figura1: Ubicación del Partido de Coronel Dorrego}

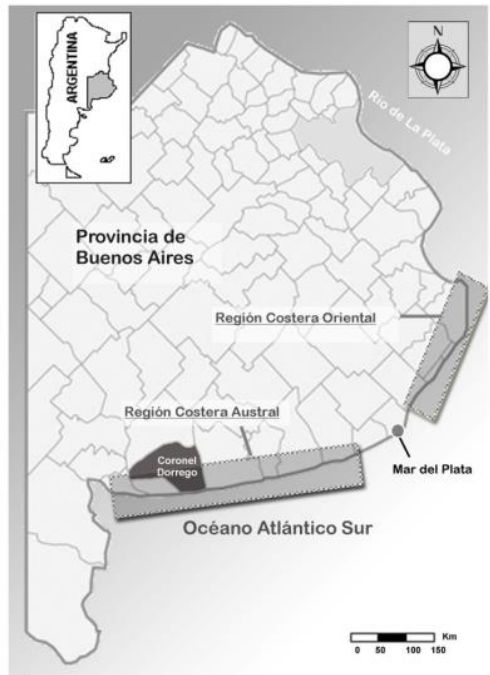

Fuente: Elaboración propia.

Ubicada de espaldas a los aproximadamente 54 kilómetros de costa atlántica dorreguense y a unos 97 kilómetros de la ciudad cabecera, Marisol se encuentra sobre el margen derecho del Río Quequén Salado. Este es la principal fuente de ingresos para la población local, muy por encima de los ingresos que generan actividades ligadas a la explotación de la costa atlántica (Municipio de Coronel Dorrego, 2011).

Al momento en que se desarrolló el conflicto, la población estable de Marisol era de tan sólo 158 habitantes (con ingresos cercanos a los de la media nacional- INDEC, 2010) y la localidad tenía una disponibilidad de parcelas vacantes del 87,93\% (Municipio de Coronel Dorrego, 2011). Para entonces, alrededor del $70 \%$ de la población económicamente activa de la 
localidad se empleaba en actividades relacionadas al turismo de pesca en el Río Quequén Salado, incluyendo la incipiente industria hotelera y gastronómica que floreció a su alrededor (Municipio de Coronel Dorrego, 2011).

Entre las organizaciones que generan algún tipo de pertenencia en Marisol pueden mencionarse dos: a la Cooperativa Marisol, encargada de la prestación del servicio de agua potable en la localidad, entre otros servicios, y al Club de Fútbol Oriente, responsable de fomentar la pesca como actividad turística y deportiva y de organizar el concurso anual de pesca deportiva en el Río Quequén Salado (el festival más popular de la localidad), con el apoyo del gobierno municipal.

La fauna del Partido de Coronel Dorrego, no poseía antecedentes de trabajos científicos relativos a su zoogeografía hasta los elaborados por integrantes de uno de los grupos en disputa, la Fundación de Historia Natural Félix de Azara (de aquí en más FA), entre los años 2005 y 2008. La FA es una organización sin fines de lucro fundada en el año 2000 , que "investiga, explora, gestiona, descubre, divulga, educa y conserva el medio ambiente" (Fundación de Historia Natural Félix de Azara, 2009: 1) a través del Departamento de Ciencias Naturales y Antropológicas de la Universidad Maimónides. Los estudios que llevó a cabo la FA, describen a las playas de Coronel Dorrego como una zona compuesta por amplios campos de dunas y gran diversidad de fauna, contando a especies de alto valor de conservación, actualmente amenazadas de extinción, como por ejemplo: la lagartija de los médanos (Liolaemus multimaculatus), exclusiva de la costa argentina, la Strunella defilippi (loica pampeana) y especies endémicas de la región pampeana, como la Asthenes hudsoni (esportillero pampeano - Fundación de Historia Natural Félix de Azara, 2005 y 2009: 12).

Según los mismos análisis, el sitio más diverso y más rico en especies vegetales de la Provincia de Buenos Aires es la costa de Coronel Dorrego. Allí, integrantes de FA identificaron 94 plantas vasculares, distribuidas en 28 familias, con un 16\% de especies exóticas (Fundación de Historia Natural Félix de Azara, 2005). El paisaje fue descrito como predominantemente ocupado por especies de herbáceas (80\%); que incluyen especies vegetales endémicas del sector comprendido entre las provincias argentinas de Buenos Aires y Chubut (se trata de dos arbustos pequeños: Senecio bergii y Baccharis divaricada -Fundación de Historia Natural Félix de Azara, 2009).

\section{El conflicto entre expertos}

Desde un primer momento, el objetivo de los estudios elaborados por la FA a lo largo de la costa atlántica bonaerense consistió en la detección de áreas prioritarias para su conservación (Fundación de Historia Natural Félix de Azara, 2005). Considerado el valor ecológico singular de la costa de Marisol, la FA junto a personal de la Dirección de Áreas Protegidas del Organismo Provincial de Desarrollo Sustentable de la Provincia de Buenos Aires (máxima autoridad ambiental de la provincia- de aquí en más OPDS), avanzó sobre una 
propuesta de reserva natural en una franja de la costa, cuya superficie terrestre se compone en un $77 \%$ de campos de dunas de entre 5 y 8 kilómetros de extensión (ver Figura 2) entre el mar y el continente (Fundación de Historia Natural Félix de Azara, 2008).

\section{Figura 2: Campo de dunas de Coronel Dorrego}

Fuente: Urquiza, 2008a

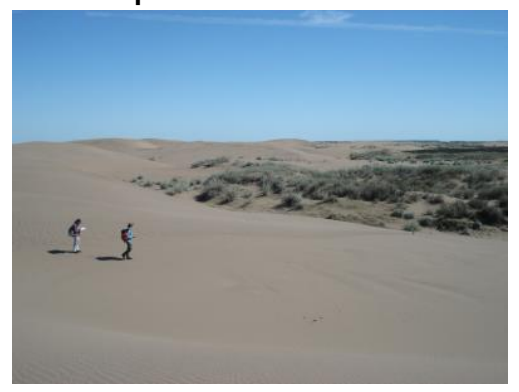

La efectivización de dicho proyecto requería de tres instancias: 1) la aprobación de la OPDS; 2) un cambio en el código de zonificación que redefiniera los usos permitidos del suelo para declarar al área reserva natural por parte del poder legislativo local; y 3) su aprobación final por parte de la Jefatura de Gabinete de la Provincia de Buenos Aires.

La primera instancia fue completada sin inconvenientes a mediados del año 2007. No obstante, mientras el proyecto promovido por la FA esperaba por la ejecución de la segunda instancia, otra coalición de expertos compuesto por integrantes de la Agencia de Cooperación Internacional del Japón (de aquí en más JICA), la Secretaría de Ambiente y Desarrollo Sustentable (máxima autoridad ambiental de la Nación Argentina -de aquí en más SAyDS) y el Área de Cambio Climático del OPDS avanzaba en la formulación de un proyecto de forestación y reforestación encuadrado dentro de los MDL (Naciones Unidas 1998).

Los proyectos MDL tienen por objeto el secuestro de dióxido de carbono mediante proyectos de forestación en el territorio de países en desarrollo financiados en su totalidad por países desarrollados. A cambio, los últimos deducen de sus promedios de emisión de gases invernaderos el aporte que dichos proyectos hagan a la mitigación del cambio climático (Naciones Unidas, 1998).

La JICA, organización promotora del proyecto MDL, es un organismo dependiente del Gobierno del Japón que ejecuta distintos programas de cooperación técnica en Argentina desde 1979, año en que se firmara el primer convenio entre ambos países. Dicho convenio fue ratificado posteriormente por ley nacional en 1981. Las acciones de la JICA en el país se encuentran focalizadas en cuatro áreas, entre las cuáles se destaca la de preservación del medio 
ambiente. Mediante ésta, la JICA actúa para "la prevención y remediación de la contaminación del aire, suelo y agua; la preservación de los ambientes naturales; las medidas contra el Calentamiento Global y la destrucción de la capa de ozono" (JICA, 2011: 1). A su vez, el área ambiental contiene específicamente cuatro proyectos, entre los que se encuentra el de "Fortalecimiento de tecnologías para la aplicación del Mecanismo de Desarrollo Limpio (MDL) de la forestación y la reforestación en la República Argentina" (JICA, 2011: 1).

El proyecto MDL mencionado, en particular, tenía por objetivos principales los de "incrementar las actividades de forestación y reforestación en el país y fortalecer las capacidades para la asistencia de los organismos de proyectos de forestación y reforestación del MDL" (JICA, 2008a: 2). La zona elegida para llevar a cabo el proyecto MDL era descrita del siguiente modo:

(...) un área costera de aproximadamente $30.000 \mathrm{Ha}$, ubicada en el partido de Coronel Dorrego, Provincia de Buenos Aires, que reviste gran importancia como ecosistema dado que sus costas son vírgenes y que es de interés proteger su playa, flora y fauna, buscando sumar así los beneficios sociales y ambientales para la comunidad local, tanto por la protección del medio como para las actividades de forestación (JICA, 2008a: 2)

Dicha zona comprendía a la franja costera considerada por la FA para ser reserva natural (JICA, 2008a; 2008b). Ante el desconocimiento mutuo de ambos grupos de expertos (incluyendo a la Dirección de Bosques de la OPDS que promoviera y aprobara el proyecto de reserva), el proyecto MDL fue aprobado por la OPDS (más específicamente por su Dirección de Cambio Climático) a fines del año 2008. De ese modo, dos proyectos mutuamente excluyentes eran aprobados por la máxima autoridad ambiental de la Provincia de Buenos Aires.

El primero de ellos, conservacionista, procuraba proteger la fauna y flora del lugar, tras considerarla de alto valor ecológico; el segundo proyecto, por su parte, procuraba "reforestar" con el fin de secuestrar emisiones de dióxido de carbono mediante la plantación de plantas vasculares pináceas -coloquialmente conocidas como Pinos- en detrimento de la flora y fauna de la zona (Besana y Monserrat, 2013; Besana, 2018). Del siguiente modo, manifestaba su sorpresa personal técnico de la propia OPDS ante la aprobación de ambos proyectos en una misma zona por parte de dos direcciones del organismo:

...era un bochorno, dos direcciones de la ODPS habían aprobado cada uno de los proyectos y los apoyaban $¡ Y$ la verdad es que los objetivos de uno contradicen a los del otro! (...) si forestas modificas el ambiente de una manera absoluta, no lo conservas (...) siempre se trata de pensar los proyectos MDL en zonas sin valor ecológico; y si tiene valor ecológico no podéis andar plantando pinos (...) porque, además, dónde plantas pinos no crece más ninguna otra planta (...) encima fijas las dunas móviles, modificando el ecosistema de muchos de los animales en peligro de extinción (Personal técnico de OPDS 2013, comunicación personal) 
Por su parte, personal de SAyDS relacionaba el intento de implementar un MDL en la zona con dos elementos: 1) el financiamiento que viene atado a éste y 2) ciertas características del ambiente:

El tema con los proyectos MDL's es que vienen atados a un fuerte financiamiento. Esto hace que a veces haya cierta premura en implementarlos. En este caso, el espacio no fue bien elegido, pero generalmente se eligen espacios de esas características... de difícil acceso, que no reporten mucho interés, ni sean necesariamente bellos (Personal técnico de SAyDS 2014, comunicación personal)

Asimismo, un informante clave arriesga una hipótesis que relaciona la impronta de la Subsecretaría de Cambio Climático y Desarrollo Sustentable de la SAyDS, una de las encargadas del proyecto, con la premura de la agencia por hacerse del financiamiento del MDL:

No es casual, yo creo... la Subsecretaría de Cambio Climático fue creada, así como la propia SAyDS y tantas otras ventanas del Estado, para capturar el financiamiento provisto por organismos internacionales en el tema ambiental (...) En los 1990, salía una línea de financiamiento y se creaba un área para capturarla... O viceversa, se obtenían financiamientos para -entre otras cosas- institucionalizar un área o temática de la agenda ambiental internacional (...) cambio climático es una de ellas (ex director general de OPDS 2016, comunicación personal)

Como puede observarse, dicha hipótesis es consistente con el argumento planteado en la tercera sección: que la agenda ambiental global influyó en el modo en que se institucionalizaron las agencias ambientales en la esfera del Estado (Gutiérrez e Isuani, 2014). No obstante, no es suficiente para explicar el conflicto entre la coalición promotora del MDL y la promotora del ANP, ambas con profesionales de agencias ambientales del Estado.

Dicho conflicto fue analizado en profundidad en otros trabajos (Besana y Monserrat, 2013; Besana, 2018). Allí se observó que las coaliciones en disputa se conformaron en torno a líneas argumentales (Hajer, 1997) que presentan ciertas similitudes y complementariedades en el modo de observar al ambiente in abstracto, pero ponderan al espacio en disputa de formas de distintas y mutuamente excluyentes. En ese sentido, mientras ambas coinciden en sus conceptos sobre la existencia de una crisis ambiental y la importancia del desarrollo sostenible (cualquiera sea el significado que le atribuya), difieren en el valor que asignan al espacio en disputa, en relación al énfasis que ponen en las relaciones desarrollo económico/conservación, ser humano/naturaleza y escala global/escala local (Besana y Monserrat, 2013 y Besana, 2018).

Por un lado, la coalición promotora del MDL, afín a un discurso conocido como Modernización Ecológica (Harvey, 1996; Harjer, 1997), ponderaba las posibilidades de generar cierto desarrollo económico en un territorio considerado de escaso valor de conservación a partir de actividades de forestación y reforestación que atienden a una problemática ambiental global. Por otro lado, la coalición promotora del ANP, afín a un discurso conocido como Ecología de la Conservación (Pullin, 2000; Onna et al., 2009), consideraba a dicho territorio como un espacio 
de alto valor de conservación, fundamental para el ecosistema local, y desaconsejaba cualquier actividad productiva o de otra índole que modifique sus características (Besana y Monserrat, 2013; Besana, 2018).

Según testimonios de los involucrados, recién a principios del año 2009, integrantes de ambas coaliciones tomaron conocimiento del proyecto de la coalición rival. Tras una serie de conversaciones y desacuerdos que duraron casi seis meses, un documento firmado por integrantes de la FA y de la JICA da cuenta de una reunión que mantuvieran ambos grupos el día 5 de septiembre de 2009. En dicho documento ambas partes acuerdan en una serie de puntos: 1) que el proyecto MDL lleve adelante estudios de elegibilidad con exclusión del área propuesta para ser declarada reserva natural; 2) que se tenga en cuenta la información de base generada por la FA para la ejecución de los estudios de elegibilidad y 3) que ninguna de las partes realice declaraciones referentes a los proyectos hasta la finalización de dichos estudios. De los tres puntos citados, el último fue el único en transgredirse. No obstante, autoridades municipales que se encontraban presentes en la reunión, apenas un día después de realizada, informaron a la prensa local lo siguiente:

(...) la realización del estudio de elegibilidad no implica que se vayan a realizar actividades de forestación y reforestación (...) el estudio incluye una instancia de taller cuyo objetivo es la búsqueda de consenso para el avance del mismo (...) es decir, reunidos diferentes sectores manifestarán sus acuerdos y objeciones que marcarán en definitiva la continuidad o no del proyecto (Suite101, 2010).

Cuatro días más tarde, la FA violaba su acuerdo con la JICA emitiendo el siguiente comunicado:

El proyecto de forestación del Organismo Provincial para el Desarrollo Sostenible tendrá, de concretarse, consecuencias ambientales extremadamente negativas, provocando en el área de inserción alteraciones en la biodiversidad local, en la dinámica hídrica, en los procesos de erosión edáfica y en la calidad del paisaje regional. Y finalmente desencadenará en la degradación y desaparición del área natural (La voz del Pueblo, 2010).

Pese a ello, el resto de los acuerdos fueron cumplidos por las partes. El estudio de elegibilidad fue entregado en noviembre del 2009. Según equipo técnico de la FA e integrantes de la OPDS, la consultora contratada para dicho trabajo realizó un relevamiento de especies animales (específicamente reptiles) y un mapeo geomorfológico; pero, en cuanto a la vegetación, no elaboró información propia, sino que copió textualmente los trabajos elaborados por la primera. En consecuencia, la consultora coincidió con la FA desestimando en última instancia la ejecución del proyecto MDL por considerar a la zona en cuestión de alto valor ecológico (JICA, 2011). Sin embargo, el proyecto no fue descartado oficialmente sino hasta el 26 de febrero del 2010, cuando SAyDS comunicó desde su página virtual lo siguiente: 
Concluyó el Proyecto de Cooperación para fortalecer el MDL Forestal entre Argentina y Japón (...) En un principio se iba a realizar en Coronel Dorrego (...) pero, finalmente, tras realizar evaluaciones de elegibilidad del terreno, de impacto y de vulnerabilidad ambiental, fue descartada. El motivo, acordado con el OPDS, fue el conflicto que se generó entre la presencia de un área de conservación y las características del MDL (SAyDS, 2010).

Por su parte, consultadas por un medio de prensa local, autoridades del JICA confirmaron la última versión, aduciendo motivos similares:

La cancelación del proyecto respondió a la controversia suscitada con algunas organizaciones no gubernamentales sobre la importancia de la zona como área natural a proteger (La voz del Pueblo, 2010).

Finalmente, el 10 de mayo de 2011, mediante el Decreto 469, el departamento de Jefatura de Gabinete de la Provincia de Buenos Aires aprueba el proyecto de reserva natural impulsado por la FA. No obstante, éste incluye sólo una parte del área considerada originalmente (Buenos Aires, 2011). Según documentos de la propia FA, la más cercana al pueblo y de menor valor ecológico (Fundación de Historia Natural Félix de Azara, 2009: 3).

Consultado sobre la aprobación de un proyecto que no satisfizo los lineamientos originales de ninguno de los proyectos propuestos por las coaliciones en conflicto un funcionario de la OPDS, señaló la siguiente:

...Yo creo que tuvo que ver que con que no hubo presión de la gente de Marisol (...) si no hay dinero mediante, cuando uno de estos conflictos se resuelve para un lado 0 para el otro es porque hay una fuerte presión de los vecinos (...) eso inclina la balanza para un lado (...) que la gente de Marisol en su mayoría no conozca el ambiente o no le interese en lo más mínimo hizo que se aprobara algo por compromiso, básicamente porque había sido un error de coordinación enorme, un escándalo (...) pero nada tenía que ver con los proyectos originales (Funcionario ODPS 2016, comunicación personal) De estar en lo cierto, la sentencia de dicho funcionario reforzaría conclusiones de estudios que consideran a la participación activa de sectores de la población local como un elemento necesario, aunque no suficiente, para que un grupo prevalezca sobre otro en un conflicto ambiental (Eg. Christel, 2016). Esté o no acertado en sus sospechas, lo cierto es que dicho funcionario insinúa una serie de cuestiones relacionadas con lo planteado en este trabajo: 1) que la mayoría de los conflictos ambientales suelen contar con un fuerte involucramiento de la población local; 2) que el caso analizado es una excepción a la regla y 3) que ello se relaciona con el desconocimiento o la falta de interés de parte de dicha población para con el ambiente de dunas. El apartado siguiente desarrolla en profundidad la vinculación entre las cuestiones mencionadas. 


\section{Discusión: ¿Un ambiente invisible?}

Aunque no es lo más común en las ciencias sociales, existen estudios que analizan no hechos sociales, no decisiones o no movilizaciones (Cf. Crenson, 1971; Auyero y Swistun, 2007). Más comunes son los trabajos que estudian casos negativos (Mahoney y Goertz, 2006). Ambos tipos de estudios comparten algunas características, por ejemplo, la importancia que en ellos adquiere la noción de principio de posibilidad. Esta señala que sólo deben ser considerados aquellos casos donde las restantes variables indican que el resultado de interés para la investigación puede ocurrir (Cf. Mahoney y Goertz, 2006). Aunque el principio de posibilidad es un recurso importante a la hora de seleccionar el o los casos de análisis, no lo es tanto a la hora de desarrollar el análisis.

En visto de ello, el presente brinda una explicación de la no participación a partir del uso de algunos elementos de la TAR, en particular los principios de simetría extendida y asociación libre (Callon, 2001; Latour, 2007). El primero propone no dar preeminencia a lo social (ni al conocimiento sociológico), sobre cualquier hecho, entidad o conocimiento de otra índole. El segundo principio propone pensar en términos de interacciones y asociaciones entre entidades humanas y no humanas (Callon, 1986).

No importa que las interacciones y asociaciones sean entre personas, objetos, animales o plantas, lo importante es que el observador identifique cómo los actores definen y asocian diversos elementos mediante los que construyen y explican el mundo, sea este calificado como natural o social (Law, 1985). Si una entidad, cualquiera sea, provoca una acción o inacción en el otro, es un elemento que puede brindar una interpretación posible de un hecho (comúnmente denominado) social (Latour, 2007). Un hecho social, en esta teoría, no es más que las representaciones, acciones y dinámicas producidas por la asociación o red entre dichas entidades humanas y no humanas.

Existen sutiles, aunque importantes, diferencias entre los autores referentes de la TAR: Latour, Law y Callon. El análisis que aquí se presenta sigue los lineamientos del último, principalmente en uno de sus artículos más conocidos, Éléments pour une sociologie de la traduction: la domestication des coquilles St. Jacques et des marins pêcheurs dans la baie de St. Brieuc (Callon, 1986). El motivo de dicha elección es metodológico: "la extensión de la simetría se realiza con prudencia" (Grossetti, 2007: 95). Desde esta perspectiva, la asociación es construida por los humanos,

(...) el observador la reconstituye sobre la base de las producciones discursivas de los humanos. Esto permite limitar a los no humanos, considerando sólo a los que fueron percibidos en producciones discursivas, y así evitar su proliferación en el análisis (Grossetti, 2007: 95).

En el estudio mencionado, Callon describió una asociación entre pescadores, vieras y científicos mediante distintas etapas, entre ellas el interesamiento (Callon, 1986: 8-9). Para el propósito del presente, dicha etapa es la única que cuenta puesto que se argumenta que no 
existió interesamiento de la población de Marisol para con el ambiente de dunas. Callon explica el término de la siguiente manera:

La etimología del término justifica su elección. Estar interesado significa estar en medio (inter-esse), estar interpuesto. Interesar a otros actores es construir mecanismos que puedan colocarse entre ellos y todas las demás entidades que quieran definir sus identidades de otra manera. A interesa a $B$ cortando o debilitando todos los lazos entre $B$ y el grupo de entidades $C, D$, E, etc., que pueden querer ligarse a $B$. Las propiedades e identidad de $B$ (ya sean vieiras, colegas o pescadores) se consolidan y/o redefinen durante el proceso de 'interesamiento'. B es el 'resultado' de la asociación que lo liga a A. Este vínculo desliga a B de todos los C's, D's y E's (si existen) que intentan darle otra definición (...) esta relación elemental empieza a dar forma y a consolidar el vínculo social (Callon, 1986: 8-9 -traducción propia).

Como se verá a continuación, que las dunas adyacentes al balneario se encuentren forestadas laxamente con tamariscos desincentiva cualquier tipo de interés de parte de los pobladores de Marisol para con el ambiente de dunas en su totalidad. Los siguientes testimonios de vecinos de la zona dan cuenta de ello:

¿Una reserva? ¿Para qué? Allá hay arena nomás y algunas malezas que traen bichos y pestes (Vecino 12009 , comunicación personal)

No suelo ir mucho, solamente hay tamariscos y arena, nada para ver (Vecino 2 2009, comunicación personal)

¡Ni son plantas! ¡Son unos pastos más feos! (Vecino 3 2009, comunicación personal)

Esos son tamariscos, sólo eso hay (Vecino 4 2011, comunicación personal)

Testimonios como los anteriores se repiten en la boca de la mayoría de los vecinos consultados. Consonantemente, cuando se les pregunta "¿Hasta dónde han recorrido la zona?" suelen responder de la siguiente manera:

No... y hasta ahí, donde terminan los tamariscos (Vecino 4 2011, comunicación personal)

Donde están los pajonales, después es toda arena (Vecino 5 2011, comunicación personal)

Y acá nomás, donde están los pastos esos (Vecino 6 2011, comunicación personal) Los pastos esos se encuentran a escasos metros del pueblo. Los otros 53 kilómetros de costa son escasamente recorridos por los pobladores. Aquellos que llevan a los turistas a pescar se acercan al Arroyo Los Gauchos (a aproximadamente 37kilómetros de Marisol), aunque por lo general manifiestan no recorrer el campo de dunas:

Mi marido va hasta donde está el arroyo, porque en temporada trabaja de eso: lleva a gente que viene a pescar (Vecino 7 2011, comunicación personal)

¿Hasta dónde voy? Ahí nomás, después es todo arena (...) no vale la pena recorrer (Vecino 8 2013, comunicación personal) 
Sólo cazadores furtivos y los dueños de los terrenos adyacentes al sector costero pueden verse, con escasa frecuencia, en la zona. Los restantes pobladores entrevistados consideran a la zona como un sector no productivo o sin valor:

Hay unos pastos ahí, arena y mugre (...) ¿Qué valor puede tener eso? (Vecino 2 2009, comunicación personal)

(...) es que no sirve para nada, no se puede plantar nada, no crece nada hay más que algunos pastizales (Vecino 8 2013, comunicación personal)

(...) lo que podía servir para algo ya lo alambraron, es de otras personas, a los pobres sólo nos dejan arena, tierra inútil (Vecino 9 2013, comunicación personal)

Las referencias al disvalor también se hacen extensibles a un plano estético (como pudo observarse en algunas de las citas anteriores), mientras que el desconocimiento sobre la fauna del lugar también parece ser generalizado.

No hay, no hay, alguna gaviota puede haber (...) pero no hay bicho que vuele o que camine por ahí (Vecino 9 2013, comunicación personal)

No me extrañaría que sólo haya ratas (Vecino 12009 , comunicación personal)

Ciertamente, estas referencias no llamaron la atención de algunos de los integrantes de la FA y ni de personal técnico de la OPDS, tal como nos refiere uno de los primeros:

(...) a pesar de que los relevamientos de flora y fauna encontraron una gran diversidad de comunidades vegetales, aves y reptiles de alto valor de conservación (...) es compresible que no les llamara la atención a los vecinos (...) a los ojos de todo aquel que no es biólogo, no son especímenes que llamen la atención. Incluso algunos de ellos, como la lagartija de los médanos, suelen ser muy escurridizos y muy chiquititos (entran en la palma de la mano) (integrante FA 2016, comunicación personal- ver Figura 3)

\section{Figura 3: Lagartija de los médanos}

Fuente: Urquiza, 2008b.

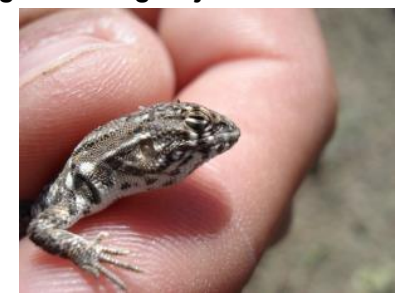

(...) es difícil llegar, nosotros íbamos en camioneta sino tenés que caminar un montonazo (...) son kilómetros y kilómetros de campos de dunas (...) eso influye en el desconocimiento que tienen los vecinos de la fauna del lugar (Integrante OPDS 2011, comunicación personal) 
En los testimonios precedentes puede observarse la falta de interesamiento (Callon, 1986) entre la población local y el ambiente de dunas. No obstante, los mismos habitantes de Marisol que no se interesaron por el ambiente de dunas sí lo hicieron por el Río Quequén Salado, evitando la instalación de una represa hidroeléctrica en su tramo inferior a mediados de los 1990 (Morel, 2016). Uno de los referentes de las protestas que lograron la sanción de una ley provincial (12.707) de protección ambiental para el Río Quequén Salado (Morel, 2016), se refiere al río de la siguiente manera:

El río es parte de mi vida y de la de todos los que crecimos acá (...) no existiría Marisol de no ser por el río, es el que nos brinda el sustento diario y la belleza que nos rodea (...) De haberse hecho la represa hoy no habría pesca, no habría sustento, en resumidas cuentas, no existiría Marisol (Vecino 14 2016, comunicación personal).

De ese modo, que no haya existido tal interesamiento (Callon, 1986) entre el ambiente de dunas y de los vecinos de Marisol -como lo hubo para con el ambiente del Río Quequén Salado- es más notorio aún, cuando se considera que los promotores de los proyectos expertos en disputa hicieron intentos por inducirlo. En ese sentido, ambos proyectos tenían entre sus ejes acciones de concientización y educación ambiental con el fin de interesar a los vecinos de Marisol y, ulteriormente, impedir su interesamiento por parte del proyecto rival. Quienes se mostraron más activos al respecto fueron los integrantes de la FA, pero como señala la siguiente entrevista el poco interesamiento que pudieron despertar en la población local se esfumó una vez hubieron abandonado el área:

No, después de que se fueron (los integrantes de la FA) no pasó nada, no hubo ningún reclamo, se desactivó totalmente. Marisol es un poblado muy chiquito, con una composición de gente muy humilde (Informante clave 2011, comunicación personal).

Sin embargo, otro interrogante puede formularse aquí: ¿Por qué las costas de Coronel Dorrego permanecieron invisibilizadas para las ciencias naturales hasta el 2008, cuando las primeras investigaciones realizadas por la FA comenzaron a ser publicadas? Este interrogante parece de importancia en la medida en que el "descubrimiento" del lugar por científicos naturales se llevó a cabo recién en 2008 , dificultando la posibilidad de que la población conociera y se interesara de antemano en el ambiente de dunas.

Una respuesta tentativa a dicho interrogante vincula (nuevamente) a algunas características del ambiente de dunas, esta vez, con el modo en que algunas ciencias han construido su conocimiento en Argentina. Históricamente el análisis en Argentina de distintos campos disciplinares como la biología, la arqueología, la geología y la paleontología entre otros, han tomado como referencia los registros de investigadores pioneros. Algunos naturalistas, como Darwin y D'Orbrigny habían realizado sus primeras observaciones a mediados del siglo XIX (Daino, 1979).

A los registros de aquellos les seguirían algunos descubrimientos de parte de Florentino Ameghino, precursor del campo de la arqueología y la paleontología en Argentina 
(Daino, 1979). Sin embargo, no existen registros de ninguno de los mencionados en la zona de Coronel Dorrego. Según los registros existentes, en 1831 Darwin llegó a recorrer el sector de la Región Costera Austral denominado "Farola de Monte Hermoso" en el actual partido de Coronel Rosales (partido lindante a Coronel Dorrego en su límite oeste) pero allí se detuvo por las dificultades que le presentaba el camino. Así se refiere a ello un experto en la zona:

Por entonces Darwin hizo una tarea extraordinaria, abarcó un gran recorrido y tomó registro de prácticamente todo (...) pero esta zona siempre fue de muy difícil acceso, lo es aún hoy (...) dudo francamente que haya llegado y si lo hizo por algún motivo no lo registró (ex director de Museo local 2012, comunicación personal).

En su exégesis histórica de la costa atlántica de Buenos Aires, Daino (1979) también relaciona la ausencia de la zona en los registros de Ameghino con las dificultades que presentaba el camino. Según aquel documento Ameghino llegó hasta el río Quequén Salado, pero la gran cantidad de dunas móviles lo desalentó a continuar por allí (Daino, 1979, p. 123).

Hayan o no arribado los investigadores pioneros a las costas de Coronel Dorrego, la falta de antecedentes históricos sobre la zona se mantuvo hasta el arribo del proyecto de la FA y las primeras publicaciones que integrantes de dicha organización realizaron. La ausencia de registros previos por parte de miembros de las ciencias naturales, confrontó a los integrantes de las FA con el desafío de lidiar con una población local que nunca había sido interesada en el ambiente de dunas, a la vez que les impidió contar con el apoyo de su propia comunidad científica. En ese sentido, la FA debió comenzar desde cero a definir el valor del área. Para cuando su tarea comenzaba a encaminarse se produjo el arribo del proyecto MDL y, posteriormente, el conflicto descrito.

\section{Reflexiones finales}

La participación de distintos actores en conflictos ambientales es objeto de una serie de debates que crecen día a día. En América Latina, existe una amplia literatura que destaca el rol que cumplen actores autóctonos o locales en el desarrollo de dichos conflictos. Desde una perspectiva macro (Vg. Gutiérrez, 2018), dicha literatura observa cómo esos actores locales se oponen a la sobreexplotación de recursos naturales por parte de empresas privadas y Estados y promueven visiones alternativas del ambiente asociadas al desarrollo de la vida diaria de sus comunidades (Eg. Gudynas y Acosta, 2011). Desde una perspectiva más micro o meso (Gutiérrez, 2018), a dicho panorama general puede agregarse otros elementos.

Para el caso particular de Argentina, por ejemplo, en este artículo se sugiere que la emergencia de conflictos ambientales no se relaciona sólo con la sobreexplotación de recursos naturales de parte de privados y/o del Estado; sino también con la impronta general con que en el último se institucionalizaron las agencias ambientales estatales en Argentina, mayormente vinculadas a la promoción de proyectos y políticas ligadas a la agenda global (Gutiérrez e Isuani, 2014). En ese sentido, aunque con menos recurrencia, dicha perspectiva global enfrenta 
resistencias provenientes del propio entramado estatal o de coaliciones que incluyen tanto a actores de la sociedad civil como estatales (Eg. Christel, 2018; Figueroa y Gutiérrez, 2018; Montera et al, 2018; Novas, 2018). El conflicto que aquí nos ocupa es ejemplo de ello.

Analizado en profundidad en otros trabajos (Besana y Monserrat, 2013; Besana, 2018), dicho conflicto opone a dos coaliciones en torno a líneas argumentales (Hajer, 1997) que presentan ciertas coincidencias y complementariedades respecto de sus consideraciones sobre el ambiente in abstracto, pero ponderan al espacio en disputa de formas de distintas y mutuamente excluyentes. En ese sentido, mientras ambas coinciden en la apelación a conceptos como crisis ambiental y desarrollo sostenible, difieren en el valor que asignan al espacio en disputa, en función del énfasis que ponen en las relaciones desarrollo económico/conservación, ser humano/naturaleza y escala global/escala local (Besana y Monserrat, 2013; Besana, 2018).

Más allá del conflicto entre expertos, este trabajo se centró en algunas de las cuestiones que lo diferencian de la mayoría de los conflictos ambientales analizados por la literatura especializada: 1) la casi inexistente participación de actores locales y 2) las características del ambiente de dunas, distintas de las de los ambientes típicamente urbanos 0 rurales que son objeto de los conflictos usualmente estudiados.

Al respecto, un análisis desde la perspectiva de la TAR (Callon, 1986), permitió ofrecer explicaciones potenciales que asocian ambas cuestiones, mediante la inclusión en él de entidades no humanas. Se argumentó que la falta de participación de la población local en el conflicto se relaciona, en gran medida, con su desconocimiento y desinterés para con el ambiente de dunas como resultado de algunas características de este último. Para dar cuenta de dicho argumento, se apeló a las representaciones y definiciones presentes en las construcciones discursivas de los actores humanos.

En ese sentido, las potenciales explicaciones reconstruidas sobre por qué no existió asociación entre los vecinos y el ambiente de dunas, referirían a características propias del último. Por un lado, la vegetación con la que los vecinos de Marisol asocian al conjunto del ambiente es, según sus testimonios, poco llamativa. Los tamariscos suelen ser llamados pastos, pastizales y son concebidos como plantas sin valor comercial o estético. Similar es lo que sucede con definiciones que hacen sobre las dunas, sólo es arena. En ellas, dicen los vecinos, no se puede plantar nada, no hay nada. En línea con dicha lógica, en esa nada misma no puede existir ser viviente alguno. Tal como reconocen los biólogos de la FA, las definiciones de los vecinos no son de extrañar. Animales como la lagartija de los médanos a pesar de tener un alto valor de conservación son invisibles a los ojos no entrenados, en este caso en particular por ser animales muy chiquitos y escurridizos.

A su vez, tal como mencionan muchos de los expertos entrevistados y algunos de los documentos analizados: la extensión del ambiente de dunas y la falta de caminos accesibles parece desanimar a quien quiera recorrer la zona, ya sea de la población local o de la comunidad científica. Esto parece haber sido un impedimento para que existieran intentos de interesamiento 
(Callon, 1986) previos al arribo de la FA que pudieran situarse entre los vecinos de Marisol y el ambiente de dunas.

En ese sentido, las definiciones y representaciones presentes en los vecinos, sin ningún otro tipo de representación con la cuál competir, influyen en la no asociación entre ambiente y comunidad local. Dicha no asociación, contrasta con las representaciones de los vecinos de Marisol para con el Río Quequén Salado y con su activa participación contra la instalación de una represa hidroeléctrica en el mismo (Morel, 2016). Al respecto, vale la pena preguntarse si la asociación entre vecinos de Marisol, por un lado, y el Río Quequén Salado, por el otro, no fue también otro elemento que se interpuso (en el sentido que le da Callon al término1986) o influyó en la no asociación entre los primeros y el ambiente de dunas.

Por último, tal como aventura un entrevistado (ver página 19), es interesante considerar como posibilidad que la ausencia de participación de sectores de la población local en el conflicto analizado no sea sólo una característica particular del mismo, sino también una variable que pudo haber incidido en un desenlace que no conformó a ninguno de las coaliciones en disputa. De ser así, en línea con otros estudios (Eg. Christel, 2016), esto podría sugerir (aunque por la negativa) que la participación activa de grupos locales en el desarrollo de conflictos ambientales, no es sólo un elemento usual en los mismos, sino también un elemento necesario o favorable para que los conflictos se resuelvan satisfactoriamente a favor de una de las posiciones enfrentadas.

\section{Referencias bibliográficas}

Acselrad, Henry. (2006). Las políticas ambientales ante las coacciones de la globalización. En Alimonda, Héctor (comp.). Los tormentos de la materia. Aportes para una ecología política latinoamericana. CLACSO. Argentina.

Alcañiz, Isabella y Gutiérrez, Ricardo A. (2009). From Local Protests to the International Court of Justice: Forging Environmental Foreign Policy in Argentina. En Harris, Paul G. (ed.).

Environmental Change and Foreign Policy. Routledge Press. Estados Unidos.

Alimonda, Héctor. (2006). Una nueva herencia en Comala [apuntes sobre la ecología política latinoamericana y la tradición marxista]. En Alimonda, Héctor (comp.). Los tormentos de la materia. Aportes para una ecología política latinoamericana. CLACSO. Argentina.

Auyero, Javier y Swistun, Deborah. (2007). Inflamable. Estudio del Sufrimiento Ambiental. Editorial Paidós. Argentina.

Azuela, Antonio. (2006). Visionarios y pragmáticos: una aproximación sociológica al derecho ambiental. UNAM/Fontamara. México.

Besana, Patricio Bruno y Monserrat, Ana Laura. (2013). Sobre arenas movedizas. Las dunas costeras como espacios del (des)conocimiento. En Merlinsky, Gabriela (comp.). Cartografías del conflicto ambiental en Argentina. CLACSO y Ediciones CICCUS. Argentina. 
Besana, Patricio Bruno. (2018). Discursos, coaliciones expertas y controversia sobre un ambiente de dunas en Buenos Aires, Argentina. Espacio Abierto. Cuaderno Venezolano de Sociología. Volumen 27, No.2. Venezuela (Pp. 209-231).

Buenos Aires. (2011). Boletín Oficial 469/11. Gobierno de la Provincia de Buenos Aires. Argentina.

Callon, Michel. (1986). Éléments pour une sociologie de la traduction: la domestication des coquilles St. Jacques et des marins pêcheurs dans la baie de St. Brieuc. L'Année Sociologique. Volumen 36, No. 1. Francia (Pp. 169 -208).

Callon, Michel. (2001). Les méthodes d'analyse des grands nombres. En Pouchet, Amélie (ed.).

Sociologie du travail: quarante ans après. Elsevier. Francia.

Christel, Lucas. (2016). Resistencias sociales y legislaciones mineras en las provincias argentinas. Los casos de Mendoza, Córdoba, Catamarca y San Juan (2003-2009).

Tesis Doctoral. Doctorado en Ciencia Política. Escuela de Política y Gobierno, Universidad Nacional de San Martín. Argentina.

Christel, Lucas. (2018). Políticas de protección ambiental para el sector minero. Entre las leyes provinciales y la Ley de Glaciares. En Gutiérrez, Ricardo (comp.). Construir el Ambiente. Sociedad, Estado y políticas ambientales en Argentina. Teseo Ediciones. Argentina.

Crenson, Mathew A. (1974). The Unpolitics of Air Pollution. A Study of Non-decision Making in the Cities. Johns Hopkins University Press. Estados Unidos.

Daino, Leonardo. (1979). Exégesis histórica de los hallazgos arqueológicos de la costa atlántica bonaerense. Prehistoria Bonaerense. Argentina.

Denzin, Norman K. y Lincoln, Yvonna S. (1994). Handbook of Qualitative Research. Sage

Publications. Estados Unidos y Gran Bretaña.

Ex director de Museo local. (2012). Comunicación personal. Buenos Aires, Argentina.

Ex director general de OPDS. (2016). Comunicación personal. Buenos Aires, Argentina.

Fernández Bouzo, Soledad. (2014). Reflexiones sobre la gestión "integrada" de cuencas hídricas en Buenos Aires. Las redes de organización social en las políticas de saneamiento ambiental. Geograficando. Volumen 10, No. 1. Extraído de: http://www.geograficando.fahce.unlp.edu.ar/article/view/5248 consultado: 23/08/2018

Figueroa, Lucas y Gutiérrez, Ricardo A. (2018). Enfrentados por el ambiente. Incidencia de las coaliciones sociedad-Estado en la protección de bosques nativos. En Gutiérrez, Ricardo (comp.). Construir el Ambiente. Sociedad, Estado y políticas ambientales en Argentina. Teseo Ediciones. Argentina.

Fischer, Frank. (2000). Citizens, Experts, and the Environment: The Politics of Local Knowledge. Duke University Press. Estados Unidos.

Funcionario ODPS. (2016). Comunicación personal. Buenos Aires, Argentina.

Fundación de Historia Natural Félix de Azara. (2005). Propuesta de Incorporación al Sistema de Áreas Naturales Protegidas de la Provincia de Buenos Aires: Reserva Natural Mixta de Objetivos Definidos 'Arroyo los Gauchos'. Fundación de Historia Natural Félix de Azara. Argentina.

Fundación de Historia Natural Félix de Azara. (2008). Vegetación dunícola de la Pampa Austral, Coronel Dorrego, Buenos Aires. Fundación de Historia Natural Félix de Azara. Argentina. 
Fundación de Historia Natural Félix de Azara. (2009). Proyecto Costas Bonaerenses. Fundación de Historia Natural Félix de Azara. Argentina.

Giarracca, Norma (comp.). (2001). ¿Una nueva ruralidad en América Latina? CLACSO. Argentina.

Glaser, Barney y Strauss, Anselm. (1967). El desarrollo de la teoría fundada. Aldine. Estados Unidos.

Grossetti, Mario. (2007). Reflexiones en torno a la noción de red. Redes. Volumen 12, No. 25. Argentina. (Pp. 85-108).

Gudynas, Eduardo y Acosta, Alberto. (2011). La renovación de la crítica al desarrollo y el buen vivir como alternativa. Utopía y Praxis Latinoamericana / Revista Internacional de Filosofía Iberoamericana y Teoría Social. Año 16. No. 53. Venezuela (Pp. 71 - 83). Gutiérrez, Ricardo A. (2015). Teoría y Praxis de los derechos ambientales en Argentina. Temas y Debates. Volumen 30. Argentina (Pp. 13-35).

Gutiérrez, Ricardo A. (2018). Introducción. En Gutiérrez, Ricardo (comp.). Construir el Ambiente. Sociedad, Estado y políticas ambientales en Argentina. Teseo Ediciones. Argentina.

Gutiérrez, Ricardo A. e Isuani, Fernando. (2014). La emergencia del ambientalismo estatal y social en Argentina. Revista de Administración Pública. Volumen 48. No. 2. Brasil (Pp. 295-322).

Hajer, Marteen A. (1997). The Politics of Enviromental Discourse. Ecological Modernization and the Policy Process. Oxford University Press. Gran Bretaña.

Hammick, Maarteen; Freeth, Dave; Koppel, Ignacio; Reeves, Steven y Barr, Homer. (2007). A best evidence systematic review of interprofessional education. Med Teach. Volumen 29, No. 8. Estados Unidos (Pp. 735-751).

Harvey, David. (1996). Justice, Nature and the Geography of Difference. Blackwell. Gran Bretaña.

INDEC. (2010). Censo Nacional 2010. INDEC. Argentina.

Informante clave. (2011). Comunicación personal. Buenos Aires, Argentina.

Integrante FA. (2016). Comunicación personal. Buenos Aires, Argentina.

Integrante OPDS. (2011). Comunicación personal. Buenos Aires, Argentina.

JICA. (2008a). Términos de Referencia para el Estudio de Elegibilidad: Proyecto de

Fomento de las Actividades de Forestación y Reforestación en el marco del MDL

Argentina. JICA. Argentina.

JICA. (2008b). Proyecto de Fomento de las Actividades de Forestación y Reforestación en el marco del MDL Argentina. JICA. Argentina.

JICA. (2011). Desarrollo de proyectos de Colaboración en Argentina. JICA. Argentina.

La Voz de Pueblo editorial. (2010). Fracasó el proyecto MDL en Coronel Dorrego. La Voz del

Pueblo. 15 de enero de 2010. Argentina (Pp. 15.).

Latour, Bruno. (2007). Reassembling the Social: An Introduction to Actor-Network-Theory. University of Oxford. Gran Bretaña y Estados Unidos.

Law, John. (1985). A Propos de Mots et des Autres Alliés. Culture Technique. Volumen 14. Francia (Pp. 58-69). 
Leff, Enrique. (2006). La ecología política en América Latina. Un campo en construcción. En Alimonda, Héctor (comp.). Los tormentos de la materia. Aportes para una ecología política latinoamericana. CLACSO. Argentina.

Mahoney, James y Goertz, Gary. (2006). The Possibility Principle: Choosing Negative Cases in Comparative Research. The American Politics Science Review. Volumen 98, No. 4. Estados Unidos (Pp. 653-669).

Martínez Allier, Joan. (1991). Ecology of the Poor: A Neglected Dimension of Latin American History. Journal of Latin American Studies. Volumen 23, No. 3. Inglaterra (Pp. 621639).

Melé, Patrice. (2006). Pour une géographie de l'action publique. Patrimoine, environnement et processus de territorialisation. Habilitation á diriger des recherches. Université Francois Rabelais. Francia.

Montera, Carolina; Moreno, Itatí; Saidon, Mariana y Verrastro, España. (2018). Las crisis como motores de cambio de las políticas de residuos. Los casos de Ciudad Autónoma de Buenos Aires y La Plata. En Gutiérrez, Ricardo (comp.). Construir el Ambiente. Sociedad, Estado y políticas ambientales en Argentina. Teseo Ediciones. Argentina.

Morel, Juan Carlos. (2016). Aplicación en la provincia de Buenos Aires de la técnica de protección del paisaje. Extraído de: https://aldiaargentina.microjuris.com/ consulta: 03/02/2017.

Municipio de Coronel Dorrego. (2011). Ciudad. Municipio de Coronel Dorrego. Argentina Naciones Unidas. (1998). Protocolo de Kyoto de la Convención Marco de las Naciones Unidas Sobre el Cambio Climático. Naciones Unidas. Territorio Internacional de las Naciones Unidas.

Novas, Mariano A. (2018). El peso de las coaliciones en las políticas de recursos naturales. La fractura hidráulica en Entre Ríos, Neuquén y Río Negro (2010-2017). En Gutiérrez, Ricardo (comp.). Construir el Ambiente. Sociedad, Estado y políticas ambientales en Argentina. Teseo Ediciones. Argentina.

Onna, Alberto, Monserrat, Ana L., Di Pasquo, Federico y Folguera, Laura. (2009). La implementación de las Áreas Naturales Protegidas entre dos tradiciones enfrentadas.

Episteme. Brasil. (Pp. 23-34).

Personal técnico de OPDS. (2013). Comunicación personal. Buenos Aires, Argentina.

Personal técnico de SAyDS. (2014). Comunicación personal. Buenos Aires, Argentina Pullin, Andrew. (2000). Conservation biology. Cambridge University Press. Reino Unido

Reboratti, Carlos. (2012). Socio-environmental Conflict in Argentina. Journal of Latin American Geography. Volumen 11, No. 2. Estados Unidos (Pp. 3-20).

Sabatini, Francisco. (1997). Conflictos ambientales y desarrollo sustentable de las regiones urbanas. Revista eure. Volumen 22, No 68. Chile (Pp. 77-91).

Sachs, Wolfgang. (1999). Sustainable Development and the Crisis of Nature: on the Politicial Anatomy of an Oxymoron. En Fischer, Frank y Hajer, Maarten A. (eds.). Living with

Nature. Environmental Politics as Cultural Discourse. Oxford University Press. Reino Unido.

Sarmiento, Domingo F. (2009). Facundo o civilización y barbarie en las pampas argentinas.

Editorial Eduvim. Argentina. 
SAyDS. (2010). Gacetillas de SAyDS. SAyDS. Argentina.

Sousa Santos, Boaventura (2001). Los nuevos movimientos sociales. OSAL. Volumen 5. (Pp. 177-188).

Suite101 editorial. (2010). Descartan la ejecución de un proyecto de forestación en el balneario Marisol. Suite101. Argentina. (Pp. 32).

Svampa, Maristella. (2012). Consenso de los commodities, giro ecoterritorial y pensamiento crítico en América Latina. OSAL. No. 32. (Pp. 15-37).

Tischler, Sergio (2004). La crisis de los canos clásicos de la forma clase y los movimientos sociales en América Latina. En Holloway, J. (comp.). Clase=Lucha. Antagonismo social y Marxismo crítico. ICSyH-BUAP/Ediciones Herramienta. México.

Tischler, Sergio (2010). La memoria ve hacia adelante. A propósito de Walter Benjamin y las nuevas rebeldías sociales. Constelaciones: Revista de Teoría Crítica. Volumen 2. España (Pp. 38-60).

Urquiza, Diego. (2008a). (Fotografía) Figura 2: Campo de dunas de Coronel Dorrego, Argentina. Urquiza, Diego. (2008b). (Fotografía) Figura 3: Lagartija de los médanos, Argentina.

Vecino 1. (2009). Comunicación personal. Buenos Aires, Argentina.

Vecino 14. (2016). Comunicación personal. Buenos Aires, Argentina.

Vecino 2. (2009). Comunicación personal. Buenos Aires, Argentina.

Vecino 3. (2009). Comunicación personal. Buenos Aires, Argentina.

Vecino 4. (2011). Comunicación personal. Buenos Aires, Argentina.

Vecino 5. (2011). Comunicación personal. Buenos Aires, Argentina.

Vecino 6. (2011). Comunicación personal. Buenos Aires, Argentina.

Vecino 7. (2011). Comunicación personal. Buenos Aires, Argentina.

Vecino 8. (2013). Comunicación personal. Buenos Aires, Argentina.

Vecino 9. (2013). Comunicación personal. Buenos Aires, Argentina. 